\title{
La enseñanza de las ciencias en la educación no formal a todos los niveles
}

\author{
Carme Zaragoza Domenech ${ }^{\mathrm{a}}$, Josep M. Fernández Novell ${ }^{\mathrm{b}, \mathrm{c}}$ \\ ${ }^{a}$ Profesora de Formación Profesional, Barcelona. España. carme.zaragoza.domenech@gmail.com \\ ${ }^{\mathrm{b}}$ Departamento de Bioquímica y Biomedicina Molecular. Universidad de Barcelona. ${ }^{\mathrm{c} C o l e g i o ~ O f i c i a l}$ \\ de Químicos de Catalunya. Barcelona. España.jmfernandeznovell@ub.edu
}

\section{Resumen}

Todo aquello que englobamos dentro de la palabra "ciencia" nos indica que cualquiera de ellas, desde la biología a la química o desde la física y la medicina hasta la ingeniería y las matemáticas, están formadas por unos conocimientos, unas metodologías y unos procesos de investigación.

Se presentarán las actividades cientificas que, utilizando la metodología de la indagación, se han realizado dentro de una "Educación no formal". Talleres y conferencias para personas adultas con poco o nulo conocimiento científico, así como juegos y experimentos preparados para niños y niñas de educación infantil y primaria. Para nuestra audiencia, la indagación implica realizar observaciones, formular preguntas, realizar experimentos, analizar e interpretar los datos obtenidos en los experimentos para después proponer explicaciones y hacer más preguntas.

En general, las actividades realizadas en los talleres para adultos y en las experiencias con los más pequeños se desarrollan de forma colaborativa, en grupos de 2 o 3 personas interactuando entre ellas, se pretende que los participantes disfruten con los experimentos cientificos. Asimismo, con los más jóvenes, es muy importante la participación, como mínimo, de una científica en la realización de los experimentos así como nombrar a los hombres y a las mujeres que han participado en la ciencia que se expone. Así se potenciará el interés científico al mostrarles personajes a seguir, especialmente en el caso de las niñas.

Estas actividades, dirigidas a segmentos distintos de nuestra sociedad, pretenden propagar la ciencia y abrir un diálogo entre la ciencia en general y dicha sociedad para corregir ciertas actitudes en contra del conocimiento científico (léase los discursos sobre antivacunas, pseudomedicinas y otros).

Palabras clave: Divulgación científica, niños, adultos, experimentos, sociedad. 


\section{Introducción}

Todo aquello que englobamos dentro de la palabra "ciencia", (la "S" de STEAM, Science, Technology, Engineering, Art and Mathematics en inglés) nos enseña que cualquiera de ellas, la biología, la química, la física, la bioquímica, la biotecnología, y otras, están formadas por conocimientos, metodologías y procesos de investigación.

Parte de nuestra sociedad tiene cierta desconfianza en la ciencia. No es nuestro objetivo ir más allá en dicha tesis, solo un par de preguntas para meditar: ¿Por qué debe importarnos tener creencias verdaderas? y ¿Por qué persisten y se propagan creencias demostrablemente falsas?. Es necesario abrir un diálogo entre la ciencia en general y nuestra sociedad para corregir las actitudes en contra de la ciencia como los discursos antivacunas, las pseudociencias y otras (visitar la plataforma Maldita.es para más información).

Para combatir estas ideas se debe contar con la participación de todas aquellas personas que están implicadas en la divulgación científica, desde los científicos y maestros hasta los alumnos y divulgadores de la ciencia. Todo ello conducirá a una alfabetización científica real (Jiménez-Pérez, 2016) de nuestra sociedad y aumentará la comprensión pública hacia la ciencia y los científicos.

Para mejorar dicho diálogo, el Colegio Oficial de Químicos de Catalunya (COQC), en colaboración con la Universidad de Barcelona (UB), ha utilizado desde hace años el aprendizaje informal tanto en los talleres dirigidos a adultos (García \& Fernández, 2019) como en aquellos destinados a los más jóvenes (Torrent \& Fernández, 2016). En este artículo se exponen las actividades realizadas desde el 2017.

En estas actividades "informales" se han desarrollado diversas aproximaciones para exponer los retos científicos del mundo actual a todos los niveles:

- Divulgar la ciencia. Presentar la ciencia y sus avances de forma comprensible.

- Promocionar la cultura científica y el uso del método científico.

- Desarrollar el espíritu crítico. Hacer que nuestra sociedad sea capaz de distinguir entre hechos demostrados científicamente y otros que no lo son.

- Alfabetización científica "real". Favorecer el fomento de la cultura científica en nuestra sociedad.

El objetivo principal es fomentar vocaciones científicas entre nuestra juventud y acercar nuestra sociedad a la ciencia, es decir, fomentar el interés por la ciencia en la ciudadanía. $\mathrm{Y}$, se desarrolla jugando y descubriendo, parafraseando a Isaac Asimov, "jugando descubrimos y jugando divulgamos estos descubrimientos". 


\section{Educación no formal}

La educación fuera de la escuela o la educación social ya fue explicada a finales del pasado siglo (Trilla, 1998). Actualmente, Marúm y Reynoso (Marúm, 2014) entienden como "educación no formal" el conjunto de procesos diferencialmente diseñados que no están directamente dirigidos a la provisión de los grados propios del Sistema Educativo Reglado y que las instituciones que la realizan no puede certificarlos para ciclos escolarizados.

La educación no formal puede tener una intencionalidad educativa y una planificación del proceso enseñanza-aprendizaje que va a ocurrir fuera del ámbito escolar.

Los puntos básicos en la educación no formal son: a) La transmisión de conocimientos y habilidades para la comunicación y la integración de la sociedad. b) Los programas dirigidos a personas distintas en edad, género, estatus social, raza, etc.

Todos los cambios en la vida familiar, el trabajo, el medio urbano, la marginación, etc., crean nuevas y distintas necesidades educativas que han de ser satisfechas por la educación no formal. Todo ello provoca, en determinadas circunstancias, un aumento de esta demanda educativa en los adultos. Además, la crisis escolar contribuye a la creación de medios, recursos e incluso instituciones no formales que complementen, suplan o sustituyan la educación convencional para el beneficio de un grupo específico de individuos.

La pandemia del COVID 19 ha mostrado la necesidad, sin precedentes, de educar a los futuros científicos, y a la población en general en entender los avances científicos y conseguir una participación de la ciudadanía que sea socialmente responsable. Además, después de la pandemia los aprendizajes no formales deberán adoptar otras formas de interacción para garantizar un enfoque sistémico de la alfabetización científica en nuestra sociedad. Es por todo ello que el COQC se ha implicado en la organización y realización de dichas actividades.

\section{Metodología}

El formato de un programa de ciencia, sea formal o informal, no puede ser una exposición académica que solo presente una relación de conceptos, ha de tener un hilo argumental que muestre el placer del descubrimiento y del entretenimiento con la ciencia.

En general, las actividades realizadas en los talleres para personas adultas se desarrollan de forma colaborativa, en grupos de 2 o 3 personas interactuando entre ellas. Mientras que los niños y niñas de primaria se dividen en grupos preparados por sus profesores o monitores. 
El trabajo en grupo (Dennick, 1998; De Vries, 2018) ha demostrado que mejora el aprendizaje porque a todos, adultos o niños, les es más fácil solucionar sus dudas o preguntas interactuando entre ellos.

Se pretende enseñar a utilizar el "Método Científico", ya que se intenta que todos los participantes desarrollen su propia iniciativa frente a las propuestas científicas, disfruten con los experimentos y las explicaciones preparadas.

Es de vital importancia la presencia de, al menos, una científica en el grupo que llevará a cabo dichas actividades. Así los participantes, sobre todo las mujeres y niñas, relacionan directamente mujeres y ciencia (Fernández-Novell, 2013). Si se quiere ampliar los horizontes científicos a las niñas hay que ir implementando estas pequeñas acciones porqué las niñas no pueden querer ser lo que no ven a su alrededor.

Las actividades se han desarrollado en Centros Cívicos que dependen de la administración municipal, Centros educativos, Colegios profesionales y otros. A continuación se muestra parte de la metodología empleada en varios ejemplos.

\subsection{Talleres y conferencias para adultos}

En el caso de la enseñanza informal para adultos solo se van a exponer, a grandes trazos, tres ejemplos de dichos talleres y conferencias, muy distintos entre sí como reflejo de la amplitud de ámbitos de dicho proyecto.

\subsubsection{Química en el laboratorio de la cocina. Centro Cívico Vil·la Urania (www).}

La cocina se convierte en un espacio de ocio y de diálogo entre los químicos/cocineros y las personas asistentes a estos talleres. En nuestra alimentación, los organismos vivos como los animales, los vegetales y los microorganismos, y los no vivos como la sal común, el bicarbonato o el aceite, todos están hechos de moléculas y de átomos, esto es química y muchas reacciones químicas ocurren en nuestra cocina. Al preparar un huevo frito de color verde a partir de caldo de col lombarda o, mientras se discute si es mejor poner la sal antes de hervir el agua o después de hervirla para hacer un caldo de carne o, incluso, cuando se discute sobre qué principios inmediatos lleva la leche natural con o sin lactosa, se van desgranando algunos conceptos químicos básicos.

\subsection{2 ¿Por qué es importante la tabla periódica? Centro Cívico Golferichs (www).}

Aprovechando que la ONU declaró el 2019 como el Año Internacional de la Tabla Periódica de los Elementos Químicos (IYPT 2019), el COQC ha diseñado unos talleres y conferencias especialmente dirigidos a dicho estudio. La Tabla Periódica de los Elementos Químicos (TP) captura la esencia no solo de la química, sino también de la astronomía, de 
la física, de la biología y de otras ciencias naturales. Es una herramienta única que permite a los científicos predecir las propiedades de la materia en la Tierra y en el Universo.

\subsubsection{Bioquímica de la diabetes, la obesidad y el cáncer. Amigos de la UNESCO (www).}

Hace solo 50 años, la diabetes, la obesidad y el cáncer eran enfermedades que producían inexorablemente la muerte de un alto porcentaje de personas. Ahora, en pleno siglo XXI y, en nuestro país, este porcentaje ha descendido notablemente gracias, en parte, a los importantes avances de la bioquímica. Estas conferencias están orientadas para que, de una forma participativa, puedan ser entendidas por personas con poca preparación científica y no relacionadas con el tema en cuestión.

\subsection{Talleres para infantil y primaria}

Para los estudiantes de infantil y primaria creemos en el juego como herramienta de aprendizaje y, utilizamos el tiempo de ocio para incorporar esta metodología. Jugar es una forma de aprender a socializarse, de transmitir valores, de investigar, de crear, de divertirse, de descubrir... la ciencia.

A los jóvenes participantes siempre se les da una bata blanca y cuando es necesario usan guantes y gafas protectoras. Les gusta vestirse como científicos en un laboratorio de verdad, y es imprescindible que se visualice la aportación femenina a la ciencia para potenciar el interés científico en todos y subrayar así la participación de las niñas. En este apartado también se van a exponer solo tres de dichos talleres con sus experimentos.

3.2.1 Ciencia en las burbujas de jabón. Feria de la ciencia en la Escuela Baloo (www).

Esta actividad se realiza durante los sábados por la mañana con niños y niñas desde P2 hasta $4^{\circ}$ de primaria. En ella se trata de interesar a los más pequeños en "descubrir", en "hacer preguntas", en "hacer ciencia" con lo que todos quieren jugar, las burbujas de jabón. Una burbuja de jabón es una película muy fina de agua jabonosa que forma una esfera llena de aire, y exhibe una superficie iridiscente con multitud de colores que permite tratar sobre el color y la luz.

\subsubsection{La física y la química en "Descubre la Ciencia" del PCB (www).}

Estos talleres, para jóvenes de 10-11 años, tienen como objetivo acercar la ciencia a dichos participantes de forma muy participativa. Con experimentos sencillos y visuales sobre física y química, que se realizan en el Openlab del PCB, se introduce el Método Científico.

Como ejemplos de actividades de física se pueden citar: "La materia y sus propiedades", "Estados de la materia" o "Electrostática". 
Como ejemplos de actividades de química se pueden citar: "Obtención de colorantes de los M\&M"; "Obtención de un indicador ácido-base de la col lombarda" o "Reacción química del bicarbonato sódico con el vinagre, el air-bag".

3.2.3 "Ciencia en familia para todos" en el Centro cívico Vil·la Uránia (www).

Esta actividad, se realiza los viernes por la tarde con 10-12 niños y niñas de 5 a 9 años acompañados de un adulto, familiar o tutor. Se trata de relacionar a los más pequeños con la ciencia mediante experimentos sencillos con la ayuda de sus familiares. Se intenta que "descubran", que "hagan preguntas" y que "jueguen con la ciencia".

Como ejemplo de los experimentos desarrolladas en estos talleres se pueden citar: "Uso de un espectroscopio" para ver la descomposición de la luz blanca, el "Peso de un globo vacío y luego lleno de aire" para responder la pregunta si el aire pesa, las "Fuerzas a distancia" usando la atracción que ejerce un globo hinchado y frotado con la bata sobre trozos de papel gracias a la electrostática, ésta se explica jugando con imanes.

\section{Resultados}

Se presentan aquellos obtenidos a partir del 2017 y en el mismo orden de los ejemplos anteriores. La Tabla 1 muestra los talleres y conferencias realizados desde el año 2017 hasta Febrero del 2020 (principio de la pandemia de COVID19), agrupados en talleres para adultos y para jóvenes. Dentro de cada conjunto están agrupados por temáticas afines.

Table 1. Talleres de educación "no formal"

\begin{tabular}{|l|l|l|l|l|l|l|}
\hline & \multicolumn{3}{|l|}{ TALLERES PARA ADULTOS } & \multicolumn{2}{l|}{ TALLERES PARA JÓVENES } \\
\hline Curso & $\begin{array}{l}\text { Ciencia y } \\
\text { cocina }\end{array}$ & $\begin{array}{l}\text { Tabla } \\
\text { periódica }\end{array}$ & $\begin{array}{l}\text { Ciencia en } \\
\text { general }\end{array}$ & $\begin{array}{l}\text { Infantil y } \\
\text { Primaria }\end{array}$ & PCB & $\begin{array}{l}\text { Ciencia } \\
\text { Familiar }\end{array}$ \\
\hline $17-18$ & 2 & - & 1 & 2 & 10 & - \\
\hline $18-19$ & 4 & 4 & 3 & 4 & 10 & 3 \\
\hline $19-20$ & 2 & 2 & 2 & 5 & 5 & 6 \\
\hline Participantes & 90 & 260 & 280 & 240 & 700 & 210 \\
\hline
\end{tabular}

\subsection{Talleres y conferencias para adultos}

Los 20 talleres para adultos se han seguido con una participación de 630 personas. 
Bajo el título de "Ciencia y cocina" se encuentran todos los relacionados con la Química en el laboratorio de la cocina donde además de exponer los conceptos químicos básicos también se ha trabajado con el frío, nitrógeno líquido y nieve carbónica o con el calor, y la cocina de baja y alta presión.

Bajo el título de "Tabla Periódica" se agrupan las conferencias ¿Por qué es importante la tabla periódica? y la síntesis espacial de los elementos químicos de la Tabla Periódica ligada con el Big Bang. Así como talleres sobre juegos de la TP.

Bajo el título "Ciencia en general" se concentran conferencias y talleres enfocados a las demandas de nuestra sociedad en temas de enfermedades (diabetes, obesidad, cáncer), nutrición, deporte, astronomía y otros.

En la Figura 1 se muestran algunos momentos de dichos talleres.

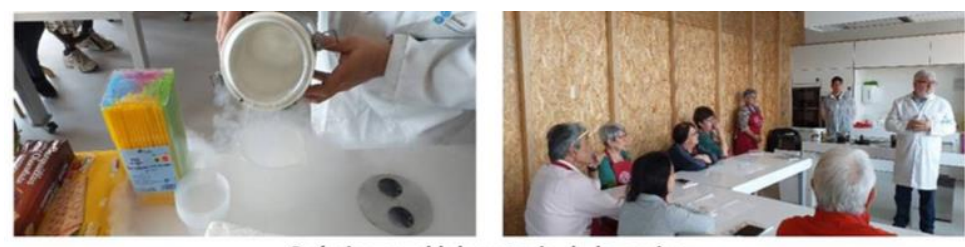

Química en el laboratorio de la cocina
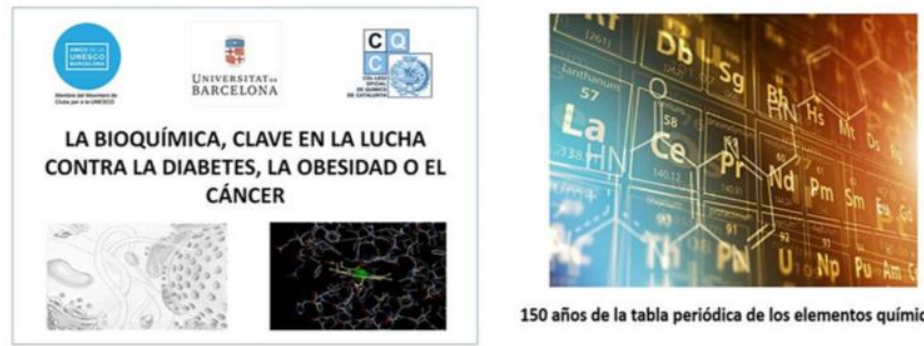

150 años de la tabla periódica de los elementos químicos

Fig. 1. En la parte superior, dos momentos del taller práctico "La química y la cocina". En la inferior, conferencias sobre la bioquímica de diversas enfermedades y la Tabla Periódica.

Tanto los Centros cívicos como las asociaciones donde se han realizado estas actividades hacen su propia evaluación. Los autores no sabemos los resultados de dicha valoración pero creemos que ésta ha sido muy positiva por la gran aceptación entre los participantes y porque ha sido necesario repetir algunos talleres con aforo limitado.

\subsection{Talleres para infantil y primaria}

Los 45 talleres para jóvenes se han seguido con una participación de 1150 niños y niñas.

Bajo el título de "Infantil y Primaria" se encuentran todos los talleres relacionados con la Ciencia básica. Algunas de las preguntas que han realizado nuestros pequeños científicos 
son: ¿De qué depende el tamaño de la burbuja, del círculo o de la fuerza del soplido?. ¿Cómo se puede mantener la burbuja en el aire sin que explote?. ¿Por qué tienen colores las burbujas?. Todas ellas acompañadas de "¿Qué pasaría si..."

Bajo el título de "PCB" se agrupan los talleres efectuados en el PCB sobre la física y la química en nuestra vida diaria. Algunas preguntas que han surgido mientras realizaban los experimentos son: ¿Por qué se separan los colores de los M\&M?. ¿De verdad que el aire pesa?. ¿Qué sustancias ácidas/básicas conoces?. Por su juventud, al igual que en los talleres anteriores, también surgen las preguntas "¿Qué pasaría si..."

Bajo el título "Ciencia Familiar" se concentran los talleres de ciencia básica para desarrollar en familia y con niños y niñas desde 5 hasta 11 años. Algunas de las preguntas que han surgido mientras realizaban los experimentos son: ¿Por qué tienen color las cosas? ¿Por qué explota un globo si lo pinchamos o acercamos una cerilla encendida?. ¿Por qué estos imanes se atraen pero estos otros no?. Todas ellas acompañadas de "¿Qué pasaría si..."

En la Figura 2 se muestran algunos momentos de dichos talleres.
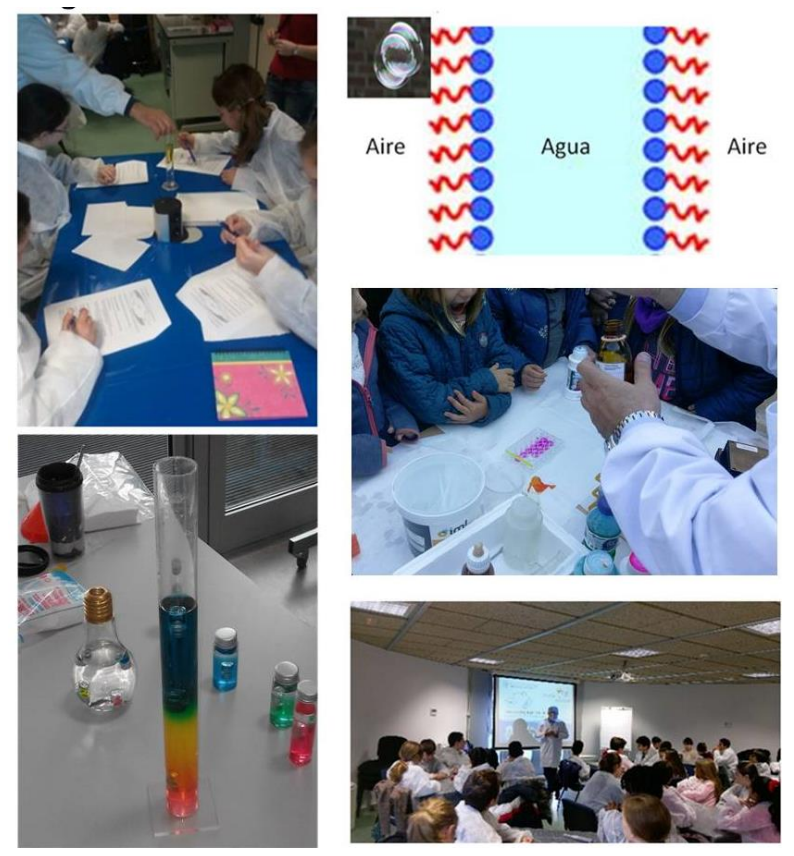

Fig. 2. Arriba a la derecha y siguiendo las agujas del reloj se observa el esquema de una burbuja de jabón, los pequeños con un familiar adulto en el patio de su escuela, el Openlab del PCB, experimentos sobre densidad y algunas guías didácticas para el alumnado.

En las actividades para infantil y primaria, son las propias entidades las que llevan a cabo su evaluación, algunas como el PCB mediante un cuestionario al profesorado del alumnado 
que realiza la actividad. Igual que en el caso de los talleres para adultos, los autores no tienen los resultados de dichas encuestas pero la valoración debe haber sido muy positiva por la gran aceptación de todas las actividades, ya que las plazas, generalmente, quedan ocupadas al completo en 2 o 3 días desde su promoción pública.

\section{Conclusiones}

Las ciencias forman parte de nuestra vida y del mundo donde vivimos. Hay una creciente preocupación sobre la falta de habilidades científicas que puede afectar a la economía (todavía no hemos dejado atrás la convulsión, científica, social y económica producida por el Covid-19). Para afrontar estos nuevos retos, presentes y futuros, necesitamos que la mayoría de la ciudadanía esté bien informada, bien formada y comprometida con todo aquello que representan las disciplinas STEAM.

La enseñanza "No Formal" de las ciencias experimentales puede y debe ayudar a afrontar dichos retos a la sociedad. En este trabajo se ha comprobado que es posible propagar ciencia entre los adultos que no están acostumbrados a relacionarse con ella. Con los más pequeños, la ciencia "No Formal" se traduce en unos juegos con la ciencia.

En ambos casos, adultos y jóvenes, la respuesta ha sido muy positiva según los propios participantes. Ello muestra el camino para conseguir una verdadera alfabetización científica en nuestra sociedad.

\section{Referencias}

Amigos UNESCO http://www.amicsunescoben.cat/ (última visita 20/03/2020)

COQC https://www.quimics.cat (última visita 10/04/2020)

De Vries, J., Tweddell, S., and McCarter, R. (2018). Team-Based Learning: Engaging learners and creating team accountability. https://uasjournal.fi/in-english/team-based-learning-engaging learners/ (última visita 16/04/2020)

Dennick, R. G., and Exley, K. (1998). Teaching and learning in groups and teams. Biochem, Education. 26, 111-115.

Escola Baloo https://agora.xtec.cat/escolabaloo/ (última visita 12/03/2020)

Fernández-Novell J.M., Zaragoza Domenech C. (2013) Women's role in science a new situation. What is being done to improve the relationship between women and science? ICERI2013 Seville, Spain, 2088-2093. 
García-Teneche, M \& Fernández-Novell, J.M. (2019) Refreshing popular science through molecular cuisine. EDULEARN19 Proceedings 11th International Conference on Education and New Learning Technologies. Palma de Mallorca, 10024-10029.

Golferichs https://www.golferichs.org/tallers-culturals/ (última visita 10/04/2020)

IYPT https://www.iypt2019.org/(última visita 26/04/2020)

Jiménez Pérez, G. E., and Tuesta, N. D. (2016). Manual de sesiones de aprendizaje con el enfoque de indagación y alfabetización científica.(VIA EIRL imprenta) Chiclayo. Perú.

Les Corts https://agora.xtec.cat/ieslescorts/(última visita 16/04/2020)

Maldita.es, https://maldita.es/malditaciencia/ (última visita 12/06/2020)

Marúm, E., and Reynoso, E. L. (2014) La importancia de la educación no formal para el desarrollo humano sustentable en México. RIES. Núm. 12, vol. V, 137-155.

PCB http://www.pcb.ub.edu/portal/ca/home (última visita 23/04/2020)

Torrent, Pol \& Fernández-Novell, J.M. (2016) Magical and chemical summer camp. Hands-on. The Heart of Science Education. Costa MF, Dorrío BV, Trna J, Trnova E (Eds.); Hands on Science Network, 91-96.

Trilla, J., Gros, B., López, F., and Martín, M. J. (2003) La educación fuera de la escuela. Ámbitos no formales y educación social. Ariel Educación, Grupo Planeta. Barcelona.

Vil·la Urània http://ajuntament.barcelona.cat/ccivics/vil.laurania (última visita 20/03/2020) 